\title{
Wetting Behaviour of Glasses on Nanostructured Silicon Surfaces
}

\author{
Sebastian Gropp ${ }^{1}$, Michael Fischer ${ }^{1}$, Lars Dittrich ${ }^{1}$, Beate Capraro ${ }^{2}$, Jens Müller ${ }^{1}$ and Martin Hoffmann ${ }^{1}$ \\ 1. Technische Universität Ilmenau, IMN MacroNano ${ }^{\circledR}$, PF 100565, Ilmenau 98694, Germany \\ 2. Fraunhofer Institute for Ceramic Technologies and Systems, Hermsdorf 1, 07629, Germany
}

\begin{abstract}
The behavior of molten glass on nanostructured silicon surface is of essential importance for the fabrication of a strong bond interface between glass (or glass-based ceramic tapes) and silicon. It was found that typical glasses do not wet the silicon surface that is always coated with a thin silica layer. It is shown that the high surface tension of molten glasses at high temperatures in combination with the dewetting surface of the structured silicon prohibits the formation of an interlocking bond between the two substrates. The theory of wetting can be applied to molten glasses, too. As a consequence, a similar solution as for liquids is investigated: the surface has to be chemically modified to become wettable. Investigations with sputtered metals on the nanostructured silicon improve wetting of the surface and result in a better bond homogeneity of the SiCer compound during sintering with low pressure.
\end{abstract}

Key words: SiCer, silicon-on-ceramics, LTCC, wetting behaviour, pressure-assisted sintering process.

\section{Introduction}

Joining silicon with glass or with glass-based materials such as low-temperature co-fired ceramics is of large interest for the assembly of microsystems. Commonly used technologies are anodic bonding or glass frit bonding. Whereas anodic bonding requires CTE-matched glasses (CTE: coefficient of thermal expansion) and extremely smooth surfaces of silicon and glass, glass frit bonding usually makes use of lead-containing solder pastes to reduce the melting point of the glass frit. Glass frit bonding is able to join even smoothly structured surface topologies, but it requires an additional material (glass frit) at the interface which is usually not perfectly matched in CTE to silicon.

In previous work it was successfully demonstrated that CTE-matched LTCC materials are suitable for a direct co-sintering with silicon. The used LTCC (low temperature cofired ceramic) green tape has a

Corresponding author: Sebastian Gropp, M.Sc., research fields: micromechanical systems and system integration. E-mail: sebastian.gropp@tu-ilmenau.de. well-adapted CTE to silicon over a wider temperature range up to more than $800{ }^{\circ} \mathrm{C}$. In this case, the glass used as binding matrix of the LTCC material develops a highly durable contact to silicon. Especially in combination with nanostructured silicon at the interface (e.g., black silicon achieved in a RIE process) results in a strong bond. In the first approach, sintering under isostatic pressure was used for a successful sintering of stress-free substrates, so-called SiCer [1, 2]. The SiCer compound substrate is ideal for RF-MEMS devices because passive RF components, e.g., coils, resistors and capacitors, can be included into the LTCC whereas the silicon part can be processed utilizing thin-film MEMS technology.

Nevertheless, this approach has also drawbacks: The sintering at $800-900{ }^{\circ} \mathrm{C}$ under high isostatic pressure causes crystal damages in the silicon. Silicon usually undergoes plastic deformations at temperatures above $600{ }^{\circ} \mathrm{C}[3]$ if a mechanical stress gradient is applied.

Although first applications could be demonstrated, the sintering at isostatic pressure limits the use of this technology. 
A careful investigation of the interface after sintering between nanostructured silicon and LTCC disclosed the key challenge: the softened glass does not wet the silicon surface; it is squeezed into the nanostructures due to the pressure, only. At edges where no pressure is applied, the glass shows a typical non-wetting shape.

For a much broader application, it is necessary to achieve wetting of the silicon by the softened glass. For this reason, here the wetting behaviour of typical glasses on nanostructured silicon is investigated.

This work is of key interest for the above-mentioned co-sintering of silicon and LTCC. It allows a bonding without use of an additional glass frit but makes use of the already existing glass phase of the LTCC.

\section{Theory and Experiments}

\subsection{Wetting Behaviour of Nanostrutured Surfaces}

For "ideal flat" surfaces, the wetting behaviour is described by Young's equation which represents the chemical surface state:

$$
\cos \theta_{y}=\left(\sigma_{s}-\sigma_{l s}\right) / \sigma_{1}
$$

With $\sigma_{1}$ and $\sigma_{s}$ for the surface of the softened LTCC and the nanostructured surface; and $\sigma_{1 s}$ interface energy between the LTCC on top of the structured silicon surface.

In 1936, Wenzel already described the effect of surface roughness on the wetting behaviour of liquids on structured solids [4]. He extended Young's law by a coefficient $r$, the so-called roughness factor. $r$ is the ratio of the structured surface area and the (projected) geometrical surface, $r \geq 1$. Wenzel's equation for the contact angle reads [5]:

$$
\cos \theta_{w}=r \cdot \cos \theta_{y}
$$

The following diagram shows Wenzel's contact angle as a function of the Young angle and the roughness factor $r$ [6]. A key result is that any kind of surface roughness intensifies the principle behaviour of a surface: wetting as well as dewetting is amplified. So if a flat surface is not wetted due to chemical reasons, a rough surface is even less wettable. With a sufficiently high pressure, this barrier can be overcome and the liquid can be squeezed into the rough surface. In this case, the gas trapped in the nanostructures can be squeezed out [7].

An investigation of the glass/silicon interface of the above-mentioned SiCer compound substrate gave rise to the assumption that the glass melt has a contact angle of below $90^{\circ}$ : In Fig. 2 a cross-sectional fracture (left) and a fracture along the interface are shown. It can clearly be seen that the silicon needles just stick in the glass matrix, they are not completely wetted. This is a well-known effect for nanostructures with dewetting properties for the liquid.

\subsection{Concept for Improving Wettability and Bondstrength}

Based on this well-known theory of wetting, it becomes clear that the contact angle between liquid (glass melt) and solid (silica due to the thin oxidation layer that always occurs on silicon, especially during sintering in air) has to be decreased by a wetting promotor to below $90^{\circ}$. The nanostructures will thus allow for a perfect wetting of the surface. On the other hand, the wetting promoter should at least not lower the adhesion of the glass on the silicon surface.

A first choice was to investigate well-known adhesion promoting layers for metals on silica: titanium or chromium, deposited by PVD in very thin layers $(<30 \mathrm{~nm})$. In this case, both materials undergo

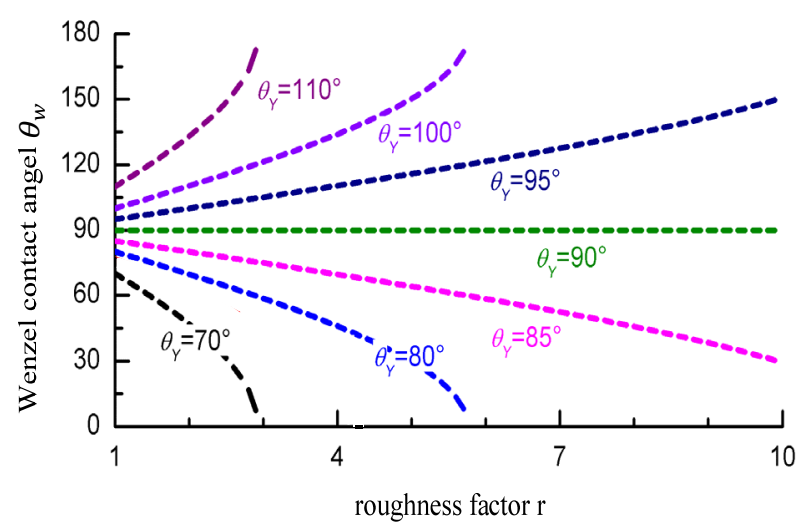

Fig. 1 Relation between Wenzel's contact angle $\theta_{w}$ and Young angle $\theta_{y}$ as a function of the roughness factor $r$. 


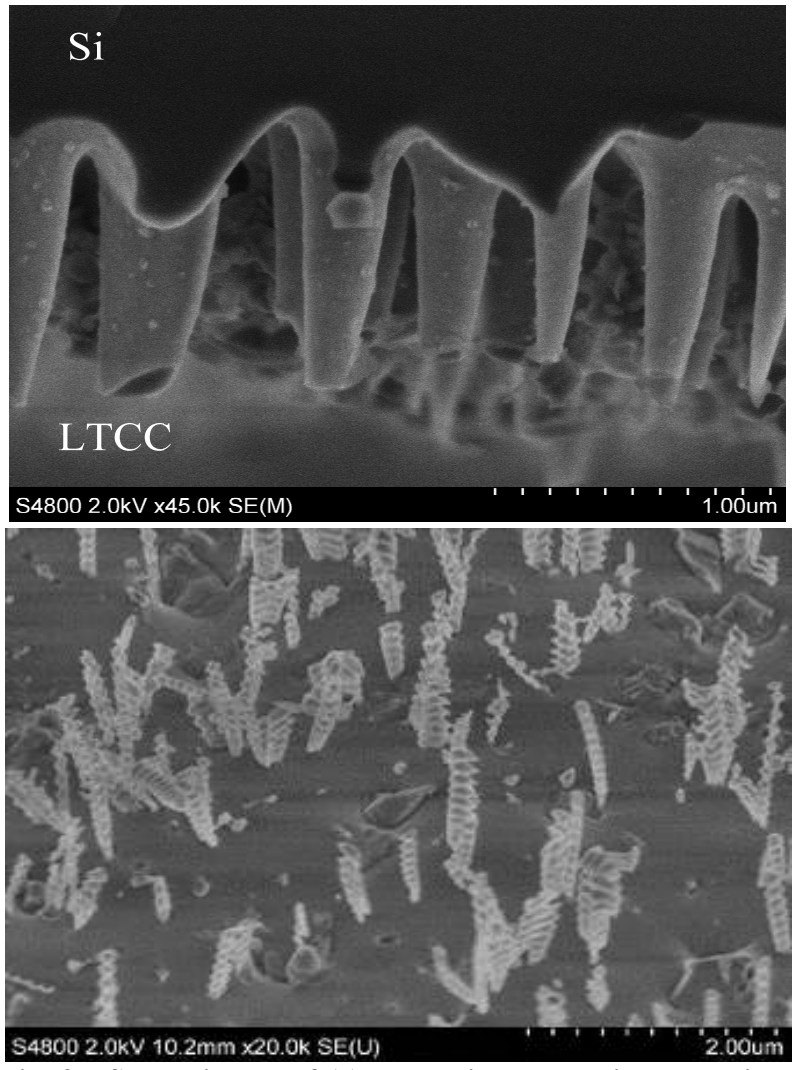

Fig. 2 SEM picture of (a) the typical dewetting behaviour between silicon nanostructures and LTCC and (b) SEM pictures of the back side of BCT-tape after sintering showing single silicon needles sticking with their tips into the molten glass matrix of the LTCC.

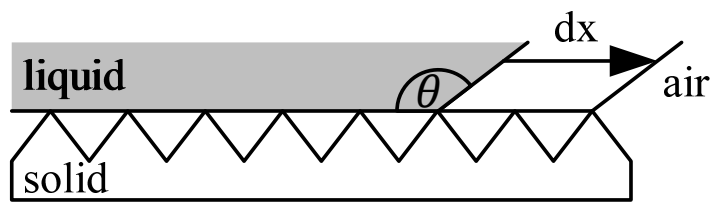

Fig. 3 Trapped air below the liquid.

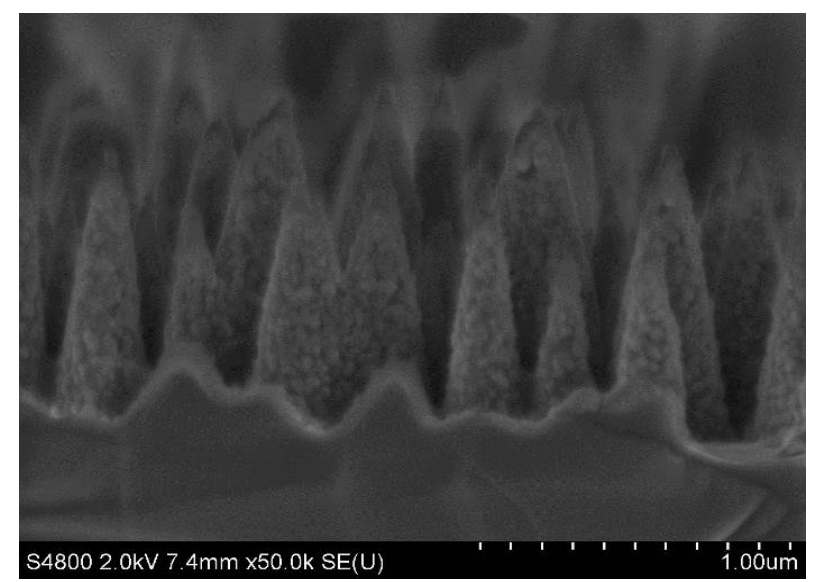

Fig. 4 SEM pictures of titanium-coated silicon needles (black silicon) after annealing in air. an oxidation during sintering as this is usually done in air. For LTCC firing, oxygen is mandatory to burn the binding polymers, too. For this reason, an oxidation of silicon and titanium can be assumed anyhow.

Fig. 3 shows a silicon nanostructure as used for SiCer fabrication, but additionally coated with titanium, after annealing in air. The surface exhibits an additional substructure of titanium dioxide in the nanometer range.

Titanium is known to be a suitable addition in low CTE glasses [8] and other glasses or glass ceramics [9] based on borosilicate glasses. It can be assumed that it forms a strong glass network close to the surface that supports wetting by the softened glass as well as an excellent bonding of the glass on the silica surface. As the total amount of titanium is rather low, it would be very difficult to identify the detailed glass composition at the interface.

\subsection{Experimental Results}

As the investigation of SiCer substrate is quite difficult after successful sintering, first tests were performed using the above-described modified silicon nanostructures and common glass powder that are also often part of the green tapes of LTCC. A finely ground powder was used for this investigation.

On silicon grass wafers $25 \mathrm{~nm}$ thin layers of chromium and titanium (Ardenne CS400 SE) were sputtered (Fig. 4). As a reference, a third wafer with uncoated silicon grass was also tested. On these wafers small amounts of B33 glass powder, a common borosilicate glass as used for anodic bonding, were applied. These samples were annealed at $900{ }^{\circ} \mathrm{C}$ for $25 \mathrm{~min}$ in air. During the sintering the titanium reacts with the oxygen of the ambient air in the furnace to titanium oxide $[10,11]$.

Table 1 shows the results of annealed B33 powder samples processed following the standard procedure in comparison with samples on which titanium was applied to the RIE silicon grass surface. The most obvious difference is the strong corona around the molten 
Table 1 Comparison molten B33 powder on black silicon and metal coated black silicon.

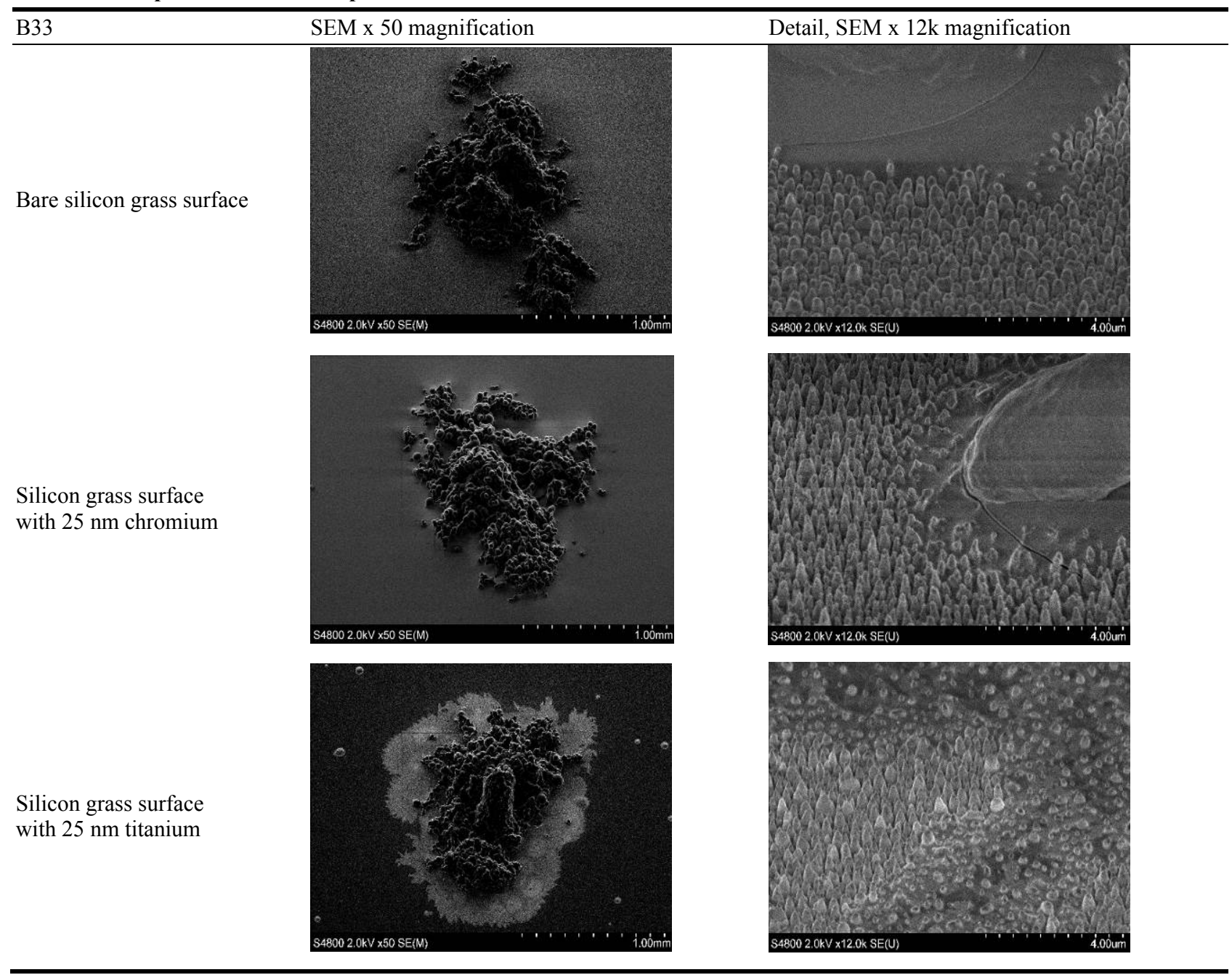

B33 powder on the $\mathrm{TiO}_{2}$ sample. A similar corona can also be found on the samples with chromium, but significantly weaker. Therefore, the following analysis focuses on the Titanium oxide samples, only.

The corona consists of solidified B33 glass that has filled the pores between the needles during annealing. Due to the fast solidification of small glass samples after sintering the triple phase contact line of hemi-wicking was identified. This type of wetting has already been discussed in Ref. [7] for common liquids. Here, the nanostructured surface is considered as porous material that "soaks" softened glass into the structures. This situation is called hemi-wicking and can be described as an intermediate state between spreading and imbibition. However, this is only a partial process and so the tips of the needles around the B33 sample are kept free from glass wetting. The principle is shown in Fig. 5. By deposition of titanium a change of the dewetting behaviour of the surface into wetting was reached. This can also be confirmed by evaluation via SEM (Fig. 6).

Finally, also tests with LTCC tapes on titanium-coated silicon have been performed. The most interesting result that was found is that the increased wetting allows reducing the isostatic pressure during sintering to almost zero. The glass matrix of the SiCer material also benefits in the same way from the wetting promoting titanium layer. Further investigations concerning an improvement of the bond strength are ongoing. First results point in this direction. 


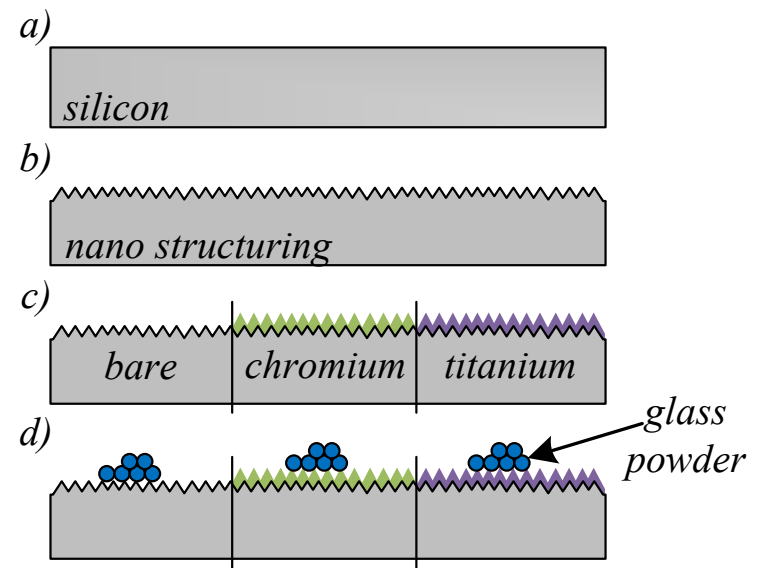

Fig. 5 Flow chart for investigation of the wetting behaviour of glass powder on bare and modified nanostructures.

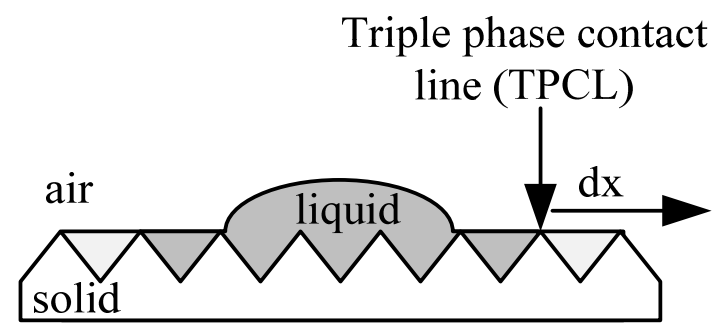

Fig. 6 Spreading and imbibition of glass in a porous material.

\section{Conclusions}

In this contribution we investigated the wetting behaviour of glass, the main matrix component of LTCC tapes, on nanostructured silicon surfaces. It was shown that untreated but oxidized silicon surfaces are almost dewetting for glasses. For this reason the surface was modified by applying a thin layer of titanium and chromium. It was found that especially titanium significantly improved the wetting behaviour of glass. Additionally, titanium is a glass-forming oxide that seems to improve the adhesion of the glass melt on silica. It could also be shown that in this case Wenzels theory for wetting on porous surfaces is also valid for the liquid "molten glass".

Future work is focused on the utilization of this effect for SiCer substrates as it allows producing compound substrates without pressure-induced crystal damage in silicon and presumably much higher bond strength.
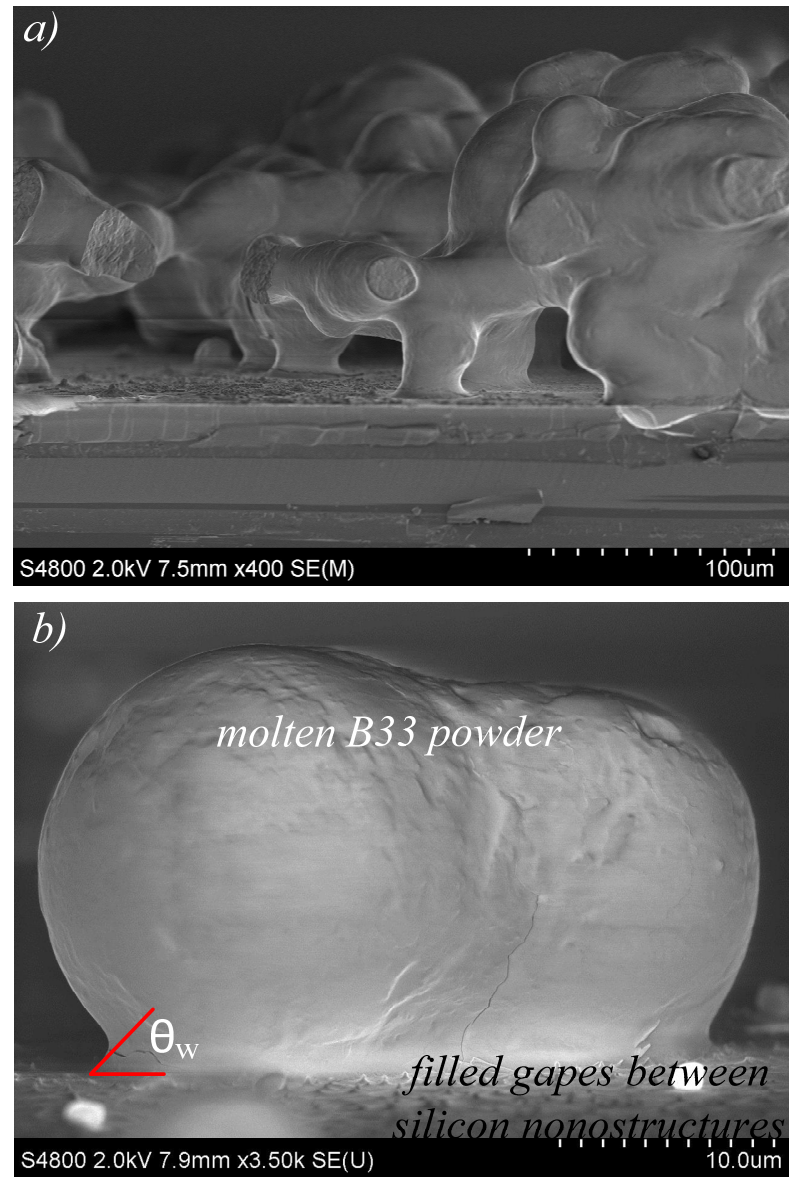

Fig. 7 SEM pictures of molten $B 33$ sample on titanium sputtered RIE nanostructures: (a) the powder forms clusters due high surface tension. In contact with the modified nanostructures, contact angle below $90^{\circ}$ could be observed; (b) detail view of on $\mathrm{B33}$ cluster in direct contact to the silicon surface.

\section{Acknowledgements}

The authors gratefully acknowledge the financial support by the DFG-German Research Foundation. FOR1522 (HO 2284/3-1, MU 3171/1-1).

\section{References}

[1] Fischer, M., Torres, H. B., Hoffmann, M., Miiller, J., PaWloWski, B., and Barth, S. 2006. Silicon ceramic composite substrate. US Patent 8,391,013 B2, filed Dec. 4, 2008, and issued Mar. 5, 2013.

[2] Fischer, M., Mache, T., Pawlowski, B., Schabbel, D., and Müller, J. 2012. "SiCer-A Substrate to Combine Ceramic and Silicon Based Micro Systems." In Proceedings of 2012 IMAPS/ACerS 8th International Conference and Exhibition on Ceramic Interconnect and Ceramic Microsystems Technologies, 158-61. 
[3] Frühauf, J., Gärtner, E., and Jänsch, E. 1999. "Silicon as a Plastic Material." Journal of Micromechanics and $\begin{array}{llll}\text { Microengineering } & 9 & \text { (4): }\end{array}$ doi:10.1088/0960-1317/9/4/304.

[4] Wenzel, R. N. 1936. "Resistance of Solid Surface to Wetting by Water." Ind. End. Chem. 28: 988-94.

[5] Quéré, D. 2005. "Non-sticking Drops.” Rep. Prog. Phys. 68: 2495-532.

[6] Leopold, S., Becker, A., Pätz, D., Knöbber, D., Ambacher, O., Sinzinger, S., and Hoffmann, M. 2012. "Needlelike Nanostructures on Aluminium Nitirde Membranes for Dynamic Lensen Using Thermo-Capillarity." Presented at MNE 2012.

[7] Bico, J., Thiele, U., and Quéré, D. 2002. "Wetting of Textured Surfaces." Colloids and Surfaces: Physicochemical and Engineering Aspects 206 (July): 41-6.

[8] ULE® Corning Code 7972, "Ultra Low Expansion Glass." http://www.corning.com/ule/.

[9] Kukharenko, S. A., Shilo, A. E., Itsenko, P. P., and Kutsai, A. N. 2010. "The Effect of Titanium Dioxide on the Structure of Silicate Multicomponent Glasses." Journal of Superhard Material 32 (6): 396-405.

[10] Ting, C., Chen, S., and Liu, D. 2000. "Structural Evolution and Optical Properties of $\mathrm{TiO}_{2}$ Thin Films Prepared by Thermal Oxidation of Sputtered Ti Films." Journal of Applied Physics 88 (8): 4628-33.

[11] Powell, O., Sweatmann, D., and Harrison, H. 2006. "The Use of Titanium and Titanium Dioxide as Masks for Deep Silicon Etching." Smart Materials and Structures 15 (1): 81-6. 